\title{
DISPROOF OF A COEFFICIENT CONJECTURE FOR MEROMORPHIC UNIVALENT FUNCTIONS
}

\author{
BY
}

ANNA TSAO

\begin{abstract}
Let $\Sigma$ denote the class of functions $g(z)=z+b_{0}+b_{1} z^{-1}+\cdots$ analytic and univalent in $|z|>1$ except for a simple pole at $\infty$. A well-known conjecture asserts that $\left|b_{n}\right| \leqslant 2 /(n+1) \quad(n=1,2, \ldots)$ with equality for $g(z)=$ $\left(1+z^{n+1}\right)^{2 /(n+1)} / z=z+2 z^{-n} /(n+1)+\cdots$. Although the conjecture is true for $n=1,2$ and certain subclasses of the class $\Sigma$, the general conjecture is known to be false for all odd $n \geqslant 3$ and $n=4$.

In $\S 2$, we generalize a variational method of Goluzin and develop second-variational techniques. This enables us in $\$ 3$ to construct explicit counterexamples to the conjecture for all $n>4$. In fact, the conjectured extremal function does not even provide a local maximum for $\operatorname{Re}\left\{b_{n}\right\}, n>4$.
\end{abstract}

1. Introduction. Let $U=\{z:|z|<1\}$ denote the unit disk and $S$ the class of functions $f(z)=z+a_{2} z^{2}+a_{3} z^{3}+\cdots$ analytic and univalent in $U$ with $f(0)=0$ and $f^{\prime}(0)=1$. An important function in $S$ is the Koebe function,

$$
k(z)=\frac{z}{(1-z)^{2}}=\sum_{n=1}^{\infty} n z^{n},
$$

which maps $U$ onto the complement of the slit from $-\frac{1}{4}$ to $\infty$ along the negative real axis.

Closely related to $S$ is the class $\Sigma$ of functions

$$
g(z)=z+b_{0}+\sum_{n=1}^{\infty} b_{n} z^{-n}
$$

analytic and univalent in $\Delta$, the exterior of the unit circle, except for a simple pole at $\infty$. Bieberbach $[2,3]$ used an elementary result due to Gronwall $[8]$ known as the area theorem to show that $\left|a_{2}\right| \leqslant 2$ for each $f \in S$, with equality if and only if $f$ is the Koebe function or one of its rotations $k_{\phi}(z)=e^{-i \phi} k\left(e^{i \phi} z\right)$.

AREA TheOREM. $\sum_{n=1}^{\infty} n\left|b_{n}\right|^{2} \leqslant 1$ for each $g \in \Sigma$.

From the extremal property of the Koebe function for $a_{2}$, Bieberbach was led to make his famous conjecture that $\left|a_{n}\right| \leqslant n$ for each $n$ and each $f \in S$.

Received by the editors August 13, 1981 and, in revised form, October 28, 1981.

1980 Mathematics Subject Classification. Primary 30C50; Secondary 30 C 70.

'This paper is part of the author's doctoral thesis written at the University of Michigan under the direction of P. L. Duren. 
The great interest and activity in the area of univalent function theory created by the Bieberbach conjecture has given rise to many related problems and conjectures. In $\$ 2$, we refine Goluzin's annular variational technique $[6,7,12,13]$ in order to consider a well-known conjecture in the class $\Sigma$.

The area theorem tells us that for each $g \in \Sigma,\left|b_{1}\right| \leqslant 1$, with extremal functions (normalized so that $b_{0}=0$ ) of the form

$$
g(z)=z+b_{1} / z, \quad\left|b_{1}\right|=1,
$$

mapping $\Delta$ onto the complement of a line segment. Schiffer [14] used a variational method to show that $\left|b_{2}\right| \leqslant \frac{2}{3}$ with equality only for

$$
g(z)=\left\{-k\left(-z^{-3}\right)\right\}^{-1 / 3}=z+\left(\frac{2}{3}\right) z^{-2}+\cdots
$$

and its rotations, which maps $\Delta$ onto the complement of three radial line segments.

On the basis of this evidence, it was conjectured that $\left|b_{n}\right| \leqslant 2 /(n+1)$ with equality for

$$
g(z)=\left\{-k\left(-z^{-n-1}\right)\right\}^{-1 /(n+1)}=z+2 z^{-n} /(n+1)+\cdots
$$

which maps $\Delta$ onto the complement of a union of $n+1$ radial line segments. The conjecture is now known to be true for certain subclasses of $\Sigma$. The analytic structure of $g$ in (1) motivates the proof of Jenkins [9] (later simplified by Duren $[5,6])$ of the conjecture for the subclass of functions in $\Sigma$ with $b_{j}=0$ for $1 \leqslant j \leqslant$ $(n-1) / 2$. Likewise, since the range of $g$ in (1) is starlike with respect to the origin, one would expect that $\left|b_{n}\right| \leqslant 2 /(n+1)$ for all starlike functions in $\Sigma$, and this has in fact been verified by Clunie [4].

In view of the strong evidence in its favor, it is surprising that the conjecture is in fact false for the full class $\Sigma$. As early as 1937, Bazilevich [1] used the Fekete-Szegö inequality to disprove the conjecture for all odd integers $n \geqslant 3$. More recently, Y. Kubota $[\mathbf{1 0 , 1 1}]$ disproved the conjecture for $n=4$.

The question remained, however, whether the conjecture is true for even indices $n \geqslant 6$. In $\S 3$, we use the expression developed in $\$ 2$ for the second variation to disprove the conjecture for every index $n>4$. We shall obtain the surprising result that the conjectured extremal function $g$ in (1) is a saddle point for $\operatorname{Re}\left\{b_{n}\right\}$ and hence does not even provide a local maximum for $\left|b_{n}\right|$. The expression we obtain for the second variation is rather complicated, but an appropriate choice of parameters simplifies it enough to permit the construction of the desired counterexamples by small perturbations of the conjectured extremal function.

2. Goluzin's annular variation. For convenience, we introduce the following notation: If a function $H(z)$ has the Laurent expansion $H(z)=\sum_{m=-\infty}^{\infty} c_{m} z^{m}$, in some annulus $r_{1}<|z|<1$, then we use $\langle H(z)\rangle_{u}$ or $\langle H\rangle_{u}$ to denote the principal part of the Laurent expansion evaluated at the point $u$; that is,

$$
\langle H(z)\rangle_{u}=\langle H\rangle_{u}=\sum_{m=-\infty}^{-1} c_{m} u^{m} .
$$


Let $f \in S$. Assume that $w^{*}=F(z, \lambda)$ is an analytic function of both $z$ and $\lambda$ in the region $r \leqslant|z|<1,|\lambda|<\lambda_{0}$, and that for each fixed $\lambda \in\left(0, \lambda_{0}\right), F$ is univalent in $r \leqslant|z|<1$. Suppose that

$$
w^{*}=F(z, \lambda)=f(z)+\lambda q(z)+O\left(\lambda^{2}\right)
$$

uniformly for $z$ in compact subsets of $r<|z|<1$ and for all sufficiently small real $\lambda$. Let $B_{\lambda}$ be the union of the image of the annulus $r \leqslant|z|<1$ with the domain interior to the image of the circle $|z|=r$ under the mapping $w^{*}=F(z, \lambda)$. For small $\lambda$, this is a simply-connected domain containing the origin. Hence there exists an analytic function $f_{\lambda}(z)$ with $f_{\lambda}(0)=0$ and $f_{\lambda}^{\prime}(0)>0$ which maps $U$ univalently onto $B_{\lambda}$. Furthermore, $f_{\lambda}(z)$ is analytic as a function of $\lambda$ for $\lambda$ sufficiently small and $z$ fixed.

Let $f_{\lambda}$ have the expansion $f_{\lambda}(z)=\sum_{m=0}^{\infty} \lambda^{m} \Phi_{m}(z)$. Goluzin [7] showed that $\Phi_{0}(z)$ $=f(z)$ and that, for $m \geqslant 1$,

$$
\Phi_{m}(z)=z f^{\prime}(z)\left[\phi_{m}(z)+\rho_{m}(z)\right],
$$

where

$$
\phi_{m}(z)=\frac{1}{m ! z f^{\prime}(z)}\left[\frac{d^{m}}{d \lambda^{m}} F\left(z \exp \left\{\sum_{k=1}^{m-1} \lambda^{k} \rho_{k}(z)\right\}, \lambda\right)\right]_{\lambda=0},
$$

and

$$
\rho_{m}(z)=\overline{\left\langle\phi_{m}\right\rangle_{1 / \bar{z}}}-\left\langle\phi_{m}\right\rangle_{z}
$$

In particular,

$$
\Phi_{1}(z)=q(z)+z f^{\prime}(z) \rho_{1}(z)
$$

Let $\theta$ be a real number and let $\alpha \in U-\{0\}$. We now choose $w^{*}=F(z, \lambda)=$ $f(z)+\lambda q(z)$, where

$$
q(z)=\frac{e^{i \theta} \alpha^{2} f^{\prime}(\alpha)^{2} f(z)^{2}}{f(\alpha)^{2}[f(z)-f(\alpha)]}
$$

Goluzin has shown that in this case,

$$
\rho_{1}(z)=\frac{e^{-i \theta} \bar{\alpha} z}{1-\bar{\alpha} z}-\frac{e^{i \theta} \alpha}{z-\alpha} .
$$

The first variational formula (5) can now be rewritten explicitly in terms of $\theta, \alpha$, and $f$. We obtain

$$
\Phi_{1}(z)=\frac{e^{i \theta} \alpha^{2} f^{\prime}(\alpha)^{2} f(z)^{2}}{f(\alpha)^{2}[f(z)-f(\alpha)]}+\frac{e^{-i \theta} \bar{\alpha} z^{2} f^{\prime}(z)}{1-\bar{\alpha} z}-\frac{e^{i \theta} \alpha z f^{\prime}(z)}{z-\alpha}
$$

Unfortunately, we cannot find $\Phi_{2}(z)$ so readily in terms of $\theta, \alpha$, and $f$. We can, however, write $\Phi_{2}(z)$ in a more explicit form by finding the derivative indicated in formula (3) with $m=2$. We thus obtain

$$
\Phi_{2}(z)=\frac{1}{2} z \rho_{1}(z)^{2}\left[f^{\prime}(z)+z f^{\prime \prime}(z)\right]+z \rho_{1}(z) q^{\prime}(z)+z f^{\prime}(z) \rho_{2}(z) .
$$


Normalizing $f_{\lambda}$ gives us a variation of the function $f$ within the class $S$. Denote this variation by $f_{\lambda}^{*}(z)$. The function $f_{\lambda}^{*}$ takes on the explicit form

$$
\begin{aligned}
f_{\lambda}^{*}(z)= & \frac{f(z)+\lambda \Phi_{1}(z)+\lambda^{2} \Phi_{2}(z)+O\left(\lambda^{3}\right)}{1+\lambda \Phi_{1}^{\prime}(0)+\lambda^{2} \Phi_{2}^{\prime}(0)+O\left(\lambda^{3}\right)} \\
= & f(z)+\lambda\left[\Phi_{1}(z)-\Phi_{1}^{\prime}(0) f(z)\right] \\
& +\lambda^{2}\left[\Phi_{2}(z)-\Phi_{1}^{\prime}(0) \Phi_{1}(z)+\Phi_{1}^{\prime}(0)^{2} f(z)-\Phi_{2}^{\prime}(0) f(z)\right]+O\left(\lambda^{3}\right) .
\end{aligned}
$$

3. Disproof of the conjecture. Henceforth, we use the convention that $\{H(z)\}_{j}$ denotes the coefficient of $z^{j}$ in the Laurent expansion of $H$ in a neighborhood of $\infty$.

We apply the variation (8) to the function

$$
f(z)=\left[g\left(z^{-1}\right)\right]^{-1}=\frac{z}{\left(z^{n+1}+1\right)^{2 /(n+1)}}=z-\frac{2}{n+1} z^{n+2}+\cdots,
$$

where $g$ is the function given by (1). The function obtained from the inversion of $f_{\lambda}^{*}$, namely,

$$
g_{\lambda}^{*}(z)=\left[f_{\lambda}^{*}\left(z^{-1}\right)\right]^{-1}=g(z)+\lambda V_{1}(z)+\lambda^{2} V_{2}(z)+O\left(\lambda^{3}\right),
$$

gives a perturbation of $g$ within the class $\Sigma$.

Let $n>4$ be a fixed integer. We will first show that

$$
\operatorname{Re}\left\{V_{1}(z)\right\}_{-n}=0 \text {. }
$$

By making judicious choices of the parameters $\theta$ and $\alpha$, we will then establish that

$$
\inf _{\substack{\alpha \in U-\{0\} \\ \theta \text { real }}} \operatorname{Re}\left\{V_{2}(z)\right\}_{-n}<0<\sup _{\substack{\alpha \in U-\{0\} \\ \theta \text { real }}} \operatorname{Re}\left\{V_{2}(z)\right\}_{-n} .
$$

It will follow immediately that for any fixed $\lambda$ sufficiently small,

$$
\inf _{\substack{\alpha \in U-\{0\} \\ \theta \text { real }}} \operatorname{Re}\left\{g_{\lambda}^{*}\right\}_{-n}<\frac{2}{n+1}<\sup _{\substack{\alpha \in U-\{0\} \\ \theta \text { real }}} \operatorname{Re}\left\{g_{\lambda}^{*}\right\}_{-n} .
$$

This will prove the following.

THEOREM. $\operatorname{Max}_{h \in \Sigma}\left|b_{n}\right|>2 /(n+1)$ for all $n>4$. In fact, the function

$$
g(z)=\left[-k\left(-z^{-n-1}\right)\right]^{-1 /(n+1)}=z+\frac{2}{n+1} z^{-n}+\cdots
$$

does not provide a local maximum for $\operatorname{Re}\left\{b_{n}\right\}, n>4$. It is actually a saddle point.

Proof. Straightforward calculation and the fact that $f\left(z^{-1}\right)=[g(z)]^{-1}$ show that in the expression (10) for $g_{\lambda}^{*}$,

$$
V_{1}(z)=\Phi_{1}^{\prime}(0) g(z)-g(z)^{2} \Phi_{1}\left(\frac{1}{z}\right)
$$

and

$$
\begin{aligned}
V_{2}(z)= & -\Phi_{1}^{\prime}(0) g(z)^{2} \Phi_{1}\left(\frac{1}{z}\right)+g(z)^{3} \Phi_{1}\left(\frac{1}{z}\right)^{2} \\
& -g(z)^{2} \Phi_{2}\left(\frac{1}{z}\right)+\Phi_{2}^{\prime}(0) g(z) .
\end{aligned}
$$


From (6), it is easily verified that $\Phi_{1}^{\prime}(0)=e^{i \theta}$. Making the substitution $\alpha=\zeta^{-1}$, we obtain from (6) and (14) that $V_{1}(z)=\sum_{k=1}^{4} S_{k}(z)$ where

$$
\begin{aligned}
& S_{1}(z)=e^{i \theta} g(z), \quad S_{2}(z)=\frac{e^{i \theta} \zeta^{2} g^{\prime}(\zeta)^{2} g(z)}{g(\zeta)[g(z)-g(\zeta)]}, \\
& S_{3}(z)=-\frac{e^{-i \theta} g^{\prime}(z)}{\bar{\zeta}\left[1-(\bar{\zeta} z)^{-1}\right]}, \quad S_{4}(z)=-\frac{e^{i \theta} z g^{\prime}(z)}{1-\zeta / z} .
\end{aligned}
$$

Henceforth, $n$ will denote a fixed integer greater than 4 . The Laurent expansions for $g(z)$ and $g^{\prime}(z)$ together with standard complex variables techniques allow us to find $\left\{S_{k}(z)\right\}_{-n}$ for $k=1,2,3,4$. We obtain

$$
\begin{aligned}
& \left\{S_{1}(z)\right\}_{-n}=2 e^{i \theta} /(n+1), \\
& \left\{S_{2}(z)\right\}_{-n}=e^{i \theta} \zeta^{2} g^{\prime}(\zeta)^{2} g(\zeta)^{n-1}, \\
& \left\{S_{3}(z)\right\}_{-n}=-e^{-i \theta} / \bar{\zeta}^{n+1},
\end{aligned}
$$

and

$$
\left\{S_{4}(z)\right\}_{-n}=2 n e^{i \theta} /(n+1)-e^{i \theta} \zeta^{n+1} .
$$

Adding the right-hand sides of equations (16)-(19), we obtain finally that

$$
\left\{V_{1}(z)\right\}_{-n}=e^{i \theta} / \zeta^{n+1}-e^{-i \theta} / \bar{\zeta}^{n+1},
$$

proving (11).

We now consider $V_{2}(z)$. In order to find an explicit form for $V_{2}(z)$, we must first apply the variational formulas (2)-(4) to the function $f$, given by (9), with $m=2$. We see that

$$
\left[\frac{d^{2}}{d \lambda^{2}} F\left(z \exp \left\{\lambda \rho_{1}(z)\right\}, \lambda\right)\right]_{\lambda=0}=z f^{\prime}(z) \rho_{1}(z)^{2}+z^{2} f^{\prime \prime}(z) \rho_{1}(z)^{2}+2 z \rho_{1}(z) q^{\prime}(z) .
$$

Hence the general formula (3) gives

$$
\phi_{2}(z)=\sum_{k=1}^{8} \psi_{k}(z)
$$

where

$$
\begin{aligned}
& \psi_{1}(z)=\frac{e^{-2 i \theta} \bar{\alpha}^{2} z^{2}}{2(1-\bar{\alpha} z)^{2}}, \quad \psi_{2}(z)=-\frac{|\alpha|^{2} z}{(1-\bar{\alpha} z)(z-\alpha)}, \\
& \psi_{3}(z)=\frac{e^{2 i \theta} \alpha^{2}}{2(z-\alpha)^{2}}, \quad \psi_{4}(z)=\frac{e^{-2 i \theta} \bar{\alpha}^{2} z^{3} f^{\prime \prime}(z)}{2 f^{\prime}(z)(1-\bar{\alpha} z)^{2}}, \\
& \psi_{5}(z)=-\frac{|\alpha|^{2} z^{2} f^{\prime \prime}(z)}{f^{\prime}(z)(1-\bar{\alpha} z)(z-\alpha)}, \quad \psi_{6}(z)=\frac{e^{2 i \theta} \alpha^{2} z f^{\prime \prime}(z)}{2 f^{\prime}(z)(z-\alpha)^{2}}, \\
& \psi_{7}(z)=\frac{|\alpha|^{2} \alpha f^{\prime}(\alpha)^{2} z f(z)[f(z)-2 f(\alpha)]}{f(\alpha)^{2}(1-\bar{\alpha} z)[f(z)-f(\alpha)]^{2}},
\end{aligned}
$$


and

$$
\psi_{8}(z)=-\frac{e^{2 i \theta} \alpha^{3} f^{\prime}(\alpha)^{2} f(z)[f(z)-2 f(\alpha)]}{f(\alpha)^{2}(z-\alpha)[f(z)-f(\alpha)]^{2}} .
$$

We would now like to find the principal part of $\psi_{k}$ for $k=1,2, \ldots, 8$. Since $\psi_{1}$ and $\psi_{4}$ are analytic in $U$, we see that $\left\langle\psi_{1}\right\rangle_{z}=\left\langle\psi_{4}\right\rangle_{z}=0$. It is readily observed that

$$
\left\langle\psi_{2}\right\rangle_{z}=-\frac{|\alpha|^{2} \alpha}{1-|\alpha|^{2}}(z-\alpha)^{-1}, \quad\left\langle\psi_{3}\right\rangle_{z}=\frac{1}{2} e^{2 i \theta} \alpha^{2}(z-\alpha)^{-2},
$$

and

$$
\left\langle\psi_{s}\right\rangle_{z}=-\frac{|\alpha|^{2} \alpha^{2} f^{\prime \prime}(\alpha)}{f^{\prime}(\alpha)\left(1-|\alpha|^{2}\right)}(z-\alpha)^{-1}
$$

We use the Taylor series expansions for $f(z)$ and $f^{\prime}(z)$ about $z=\alpha$ and standard series techniques to find $\left\langle\psi_{6}\right\rangle_{2},\left\langle\psi_{7}\right\rangle_{z}$, and $\left\langle\psi_{8}\right\rangle_{z}$. We deduce, after lengthy but straightforward calculations, that

$$
\begin{aligned}
& \left\langle\psi_{6}\right\rangle_{z}=\frac{1}{2} e^{2 i \theta} \alpha^{2}\left\{\frac{\alpha f^{\prime \prime}(\alpha)}{f^{\prime}(\alpha)}(z-\alpha)^{-2}+\left[\frac{f^{\prime \prime}(\alpha)}{f^{\prime}(\alpha)}+\frac{\alpha f^{\prime \prime \prime}(\alpha)}{f^{\prime}(\alpha)}-\frac{\alpha f^{\prime \prime}(\alpha)^{2}}{f^{\prime}(\alpha)^{2}}\right](z-\alpha)^{-1}\right\}, \\
& \left\langle\psi_{7}\right\rangle_{z}=\frac{\alpha|\alpha|^{2}}{1-|\alpha|^{2}}\left\{-\alpha(z-\alpha)^{-2}+\left[\frac{\alpha f^{\prime \prime}(\alpha)}{f^{\prime}(\alpha)}-1-\frac{|\alpha|^{2}}{1-|\alpha|^{2}}\right](z-\alpha)^{-1}\right\},
\end{aligned}
$$

and

$$
\begin{aligned}
\left\langle\psi_{8}\right\rangle_{z}=e^{2 i \theta} \alpha^{3}\left\{(z-\alpha)^{-3}-\frac{f^{\prime \prime}(\alpha)}{f^{\prime}(\alpha)}(z-\alpha)^{-2}\right. & \\
& \left.+\left[\frac{3 f^{\prime \prime}(\alpha)^{2}}{4 f^{\prime}(\alpha)^{2}}-\frac{f^{\prime \prime \prime}(\alpha)}{3 f^{\prime}(\alpha)}-\frac{f^{\prime}(\alpha)^{2}}{f(\alpha)^{2}}\right](z-\alpha)^{-1}\right\} .
\end{aligned}
$$

We are now able to calculate $\rho_{2}(z)$ explicitly. It is easily seen from equations (4) and (21) that

$$
\rho_{2}(z)=\sum_{k=1}^{8}\left[\overline{\left\langle\psi_{k}\right\rangle_{1 / z}}-\left\langle\psi_{k}\right\rangle_{z}\right]
$$

Therefore, using the expressions just obtained for the principal parts of $\psi_{1}, \ldots, \psi_{8}$, we obtain after a lengthy simplification that

$$
\rho_{2}(z)=e^{2 i \theta} A(z)+e^{-2 i \theta} B(z)+C(z),
$$


where

$$
\begin{aligned}
A(z)= & -\frac{\alpha^{3}}{(z-\alpha)^{3}}+\frac{1}{(z-\alpha)^{2}}\left[\frac{\alpha^{3} f^{\prime \prime}(\alpha)}{2 f^{\prime}(\alpha)}-\frac{\alpha^{2}}{2}\right] \\
& +\frac{1}{z-\alpha}\left[\frac{\alpha^{3} f^{\prime}(\alpha)^{2}}{f(\alpha)^{2}}-\frac{\alpha^{2} f^{\prime \prime}(\alpha)}{2 f^{\prime}(\alpha)}-\frac{\alpha^{3} f^{\prime \prime \prime}(\alpha)}{6 f^{\prime}(\alpha)}-\frac{\alpha^{3} f^{\prime \prime}(\alpha)^{2}}{4 f^{\prime}(\alpha)^{2}}\right], \\
B(z)= & \frac{\bar{\alpha}^{3} z^{3}}{(1-\bar{\alpha} z)^{3}}+\frac{z^{2}}{(1-\bar{\alpha} z)^{2}}\left[\frac{\alpha^{2}}{2}-\frac{\alpha^{3} f^{\prime \prime}(\alpha)}{2 f^{\prime}(\alpha)}\right] \\
& +\frac{z}{1-\bar{\alpha} z}\left[\frac{\alpha^{2} f^{\prime \prime}(\alpha)}{2 f^{\prime}(\alpha)}+\frac{\alpha^{3} f^{\prime \prime \prime}(\alpha)}{6 f^{\prime}(\alpha)}+\frac{\alpha^{3} f^{\prime \prime}(\alpha)^{2}}{4 f^{\prime}(\alpha)^{2}}-\frac{\alpha^{3} f^{\prime}(\alpha)^{2}}{f(\alpha)^{2}}\right],
\end{aligned}
$$

and

$$
\begin{aligned}
C(z)= & \frac{\alpha^{2}|\alpha|^{2}}{\left(1-|\alpha|^{2}\right)(z-\alpha)^{2}}+\frac{1}{z-\alpha}\left[\frac{2 \alpha|\alpha|^{2}}{1-|\alpha|^{2}}+\frac{\alpha|\alpha|^{4}}{\left(1-|\alpha|^{2}\right)^{2}}\right] \\
& -\frac{\bar{\alpha}^{2}|\alpha|^{2} z^{2}}{\left(1-|\alpha|^{2}\right)(1-\bar{\alpha} z)^{2}}-\frac{z}{1-\bar{\alpha} z}\left[\frac{2 \bar{\alpha}|\alpha|^{2}}{1-|\alpha|^{2}}+\frac{\bar{\alpha}|\alpha|^{4}}{\left(1-|\alpha|^{2}\right)^{2}}\right]
\end{aligned}
$$

Finally then, we see from equations (2), (21) and (22) that $\Phi_{2}(z)$ takes the form

$$
\Phi_{2}(z)=z f^{\prime}(z)\left[\sum_{k=1}^{8} \psi_{k}(z)+e^{2 i \theta} A(z)+e^{-2 i \theta} B(z)+C(z)\right] \text {. }
$$

We now consider the right-hand side of equation (15) term by term in order to find $\operatorname{Re}\left\{V_{2}(z)\right\}_{-n}$. Using the expression obtained in (20) for $\left\{V_{1}(z)\right\}_{-n}$, it is clear from the first-variational formula (14) that

$$
\begin{aligned}
\operatorname{Re}\left\{-\Phi_{1}^{\prime}(0) g(z)^{2} \Phi_{1}\left(\frac{1}{z}\right)\right\}_{-n} & =\operatorname{Re}\left\{\Phi_{1}^{\prime}(0) V_{1}(z)-\Phi_{1}^{\prime}(0)^{2} g(z)\right\}_{-n} \\
& =\operatorname{Re}\left\{\frac{e^{2 i \theta}}{\zeta^{n+1}}-\frac{1}{\zeta^{n+1}}-\frac{2 e^{2 i \theta}}{n+1}\right\} .
\end{aligned}
$$

It is easily verified from the expression (23) for $\Phi_{2}(z)$ and the explicit expressions given for $\psi_{k}(k=1,2, \ldots, 8), A(z), B(z)$, and $C(z)$ that

$$
\begin{aligned}
\Phi_{2}^{\prime}(0)= & \psi_{3}(0)+e^{2 i \theta} A(0)+C(0) \\
= & e^{2 i \theta}\left[1+\frac{\alpha f^{\prime \prime}(\alpha)}{f^{\prime}(\alpha)}-\frac{\alpha^{2} f^{\prime}(\alpha)^{2}}{f(\alpha)^{2}}+\frac{\alpha^{2} f^{\prime \prime \prime}(\alpha)}{6 f^{\prime}(\alpha)}+\frac{\alpha^{2} f^{\prime \prime}(\alpha)^{2}}{4 f^{\prime}(\alpha)^{2}}\right] \\
& -\frac{|\alpha|^{2}}{1-|\alpha|^{2}}-\frac{|\alpha|^{4}}{\left(1-|\alpha|^{2}\right)^{2}} .
\end{aligned}
$$


The substitution $\alpha=\zeta^{-1}$ in the expression obtained for $\Phi_{2}^{\prime}(0)$ allows us to show that

$$
\begin{aligned}
\operatorname{Re}\left\{\Phi_{2}^{\prime}(0) g(z)\right\}_{-n}=\frac{2}{n+1} \operatorname{Re}\left\{e^{2 i \theta}[1\right. & +\frac{f^{\prime \prime}(1 / \zeta)}{\zeta f^{\prime}(1 / \zeta)}-\frac{f^{\prime}(1 / \zeta)^{2}}{\zeta^{2} f(1 / \zeta)^{2}} \\
& \left.+\frac{f^{\prime \prime \prime}(1 / \zeta)}{6 \zeta^{2} f^{\prime}(1 / \zeta)}+\frac{f^{\prime \prime}(1 / \zeta)^{2}}{4 \zeta^{2} f^{\prime}(1 / \zeta)^{2}}\right] \\
& \left.-\frac{1}{|\zeta|^{2}-1}-\frac{1}{\left(|\zeta|^{2}-1\right)^{2}}\right\}
\end{aligned}
$$

Making the substitutions $\alpha=\zeta^{-1}, f\left(z^{-1}\right)=g(z)^{-1}$ and $f^{\prime}\left(z^{-1}\right)=z^{2} g^{\prime}(z) g(z)^{-2}$ in the expression for $\Phi_{1}\left(z^{-1}\right)$ obtained by substituting $z^{-1}$ for $z$ in (6), we obtain

$$
\Phi_{1}\left(\frac{1}{z}\right)=-\frac{e^{i \theta} \zeta^{2} g^{\prime}(\zeta)^{2}}{g(\zeta) g(z)[g(z)-g(\zeta)]}+\frac{e^{-i \theta} g^{\prime}(z)}{\bar{\zeta} g(z)^{2}\left[1-(\bar{\zeta} z)^{-1}\right]}+\frac{e^{i \theta} z g^{\prime}(z)}{g(z)^{2}(1-\zeta / z)},
$$

which yields the formula

$$
g(z)^{3} \Phi_{1}\left(\frac{1}{z}\right)^{2}=\sum_{k=1}^{6} T_{k}(z)
$$

where

$$
\begin{aligned}
& T_{1}(z)=\frac{e^{2 i \theta} \zeta^{4} g^{\prime}(\zeta)^{4} g(z)}{g(\zeta)^{2}[g(z)-g(\zeta)]^{2}} \\
& T_{2}(z)=-\frac{2 \zeta^{2} g^{\prime}(\zeta)^{2} g^{\prime}(z)}{\bar{\zeta} g(\zeta)[g(z)-g(\zeta)]\left[1-(\bar{\zeta} z)^{-1}\right]} \\
& T_{3}(z)=-\frac{2 e^{2 i \theta} \zeta^{2} g^{\prime}(\zeta)^{2} z g^{\prime}(z)}{g(\zeta)[g(z)-g(\zeta)](1-\zeta / z)} \\
& T_{4}(z)=\frac{e^{-2 i \theta} g^{\prime}(z)^{2}}{\bar{\zeta}^{2} g(z)\left[1-(\bar{\zeta} z)^{-1}\right]^{2}} \\
& T_{5}(z)=\frac{2 z g^{\prime}(z)^{2}}{\bar{\zeta} g(z)\left[1-(\bar{\zeta} z)^{-1}\right](1-\zeta / z)}
\end{aligned}
$$

and

$$
T_{6}(z)=\frac{e^{2 i \theta} z^{2} g^{\prime}(z)^{2}}{g(z)(1-\zeta / z)^{2}} .
$$

Techniques analogous to those described previously for finding the first variation $\left\{V_{1}(z)\right\}_{-n}$ are used to find $\operatorname{Re}\left\{T_{k}(z)\right\}_{-n}$ for $k=1,2, \ldots, 6$. We obtain

$$
\operatorname{Re}\left\{T_{1}(z)\right\}_{-n}=\operatorname{Re}\left\{n e^{2 i \theta} \zeta^{4} g^{\prime}(\zeta)^{4} g(\zeta)^{n-3}\right\},
$$




$$
\begin{gathered}
\operatorname{Re}\left\{T_{2}(z)\right\}_{-n}=-\operatorname{Re}\left\{2 \zeta^{2} g^{\prime}(\zeta)^{2} \sum_{k=0}^{n-1} \frac{g(\zeta)^{n-2-k}}{\bar{\zeta}^{k+1}}\right\}, \\
\operatorname{Re}\left\{T_{3}(z)\right\}_{-n}=-\operatorname{Re}\left\{2 e^{2 i \theta} g^{\prime}(\zeta)^{2} \sum_{k=0}^{n} g(\zeta)^{n-k-1} \zeta^{k+2}\right\}, \\
\operatorname{Re}\left\{T_{4}(z)\right\}_{-n}=\operatorname{Re}\left\{n e^{-2 i \theta} / \bar{\zeta}^{n+1}\right\}, \\
\operatorname{Re}\left\{T_{5}(z)\right\}_{-n}=\operatorname{Re}\left\{2 \sum_{k=0}^{n} \frac{\zeta^{n-k}}{\bar{\zeta}^{k+1}}\right\},
\end{gathered}
$$

and

$$
\operatorname{Re}\left\{T_{6}(z)\right\}_{-n}=\operatorname{Re}\left\{(n+2) e^{2 i \theta} \zeta^{n+1}-(4 n+2) e^{2 i \theta} /(n+1)\right\} .
$$

The substitution $z^{-1}$ for $z$ in the expression for $\Phi_{2}(z)$ in (23) and the fact that $f^{\prime}\left(z^{-1}\right)=z^{2} g^{\prime}(z) g(z)^{-2}$ give us that

$$
-g(z)^{2} \Phi_{2}\left(\frac{1}{z}\right)=-z g^{\prime}(z)\left[\sum_{k=1}^{8} \psi_{k}\left(\frac{1}{z}\right)+e^{2 i \theta} A\left(\frac{1}{z}\right)+e^{-2 i \theta} B\left(\frac{1}{z}\right)+C\left(\frac{1}{z}\right)\right] .
$$

Straightforward calculation shows that

$$
\begin{aligned}
& -z g^{\prime}(z) \psi_{1}\left(\frac{1}{z}\right)=-\frac{e^{-2 i \theta} g^{\prime}(z)}{2 \bar{\zeta}^{2} z\left[1-(\bar{\zeta} z)^{-1}\right]^{2}}, \\
& -z g^{\prime}(z) \psi_{2}\left(\frac{1}{z}\right)=-\frac{g^{\prime}(z)}{\bar{\zeta}\left[1-(\bar{\zeta} z)^{-1}\right](1-\zeta / z)}, \\
& -z g^{\prime}(z) \psi_{3}\left(\frac{1}{z}\right)=-\frac{e^{2 i \theta} z g^{\prime}(z)}{2(1-\zeta / z)^{2}}, \\
& -z g^{\prime}(z) \psi_{4}\left(\frac{1}{z}\right)=-\frac{e^{-2 i \theta} f^{\prime \prime}(1 / z) g(z)^{2}}{2 \bar{\zeta}^{2} z^{4}\left[1-(\bar{\zeta} z)^{-1}\right]^{2}}, \\
& -z g^{\prime}(z) \psi_{5}\left(\frac{1}{z}\right)=-\frac{f^{\prime \prime}(1 / z) g(z)^{2}}{\bar{\zeta} z^{3}\left[1-(\bar{\zeta} z)^{-1}\right](1-\zeta / z)}, \\
& -z g^{\prime}(z) \psi_{6}\left(\frac{1}{z}\right)=-\frac{e^{2 i \theta} f^{\prime \prime}(1 / z) g(z)^{2}}{2 z^{2}(1-\zeta / z)^{2}} \text {, } \\
& -z g^{\prime}(z) \psi_{7}\left(\frac{1}{z}\right)=\frac{2 \zeta^{2} g^{\prime}(\zeta)^{2} g^{\prime}(z)[g(z)-g(\zeta) / 2]}{\bar{\zeta} g(\zeta)\left[1-(\bar{\zeta} z)^{-1}\right][g(z)-g(\zeta)]^{2}}, \\
& -z g^{\prime}(z) \psi_{8}\left(\frac{1}{z}\right)=\frac{2 e^{2 i \theta} \zeta^{2} g^{\prime}(\zeta)^{2} z g^{\prime}(z)[g(z)-g(\zeta) / 2]}{g(\zeta)(1-\zeta / z)[g(z)-g(\zeta)]^{2}},
\end{aligned}
$$




$$
\begin{aligned}
& -e^{2 i \theta} z g^{\prime}(z) A\left(\frac{1}{z}\right)=-e^{2 i \theta} z g^{\prime}(z)\left\{\frac{1}{(1-\zeta / z)^{3}}+\frac{1}{(1-\zeta / z)^{2}}\left[\frac{f^{\prime \prime}(1 / \zeta)}{2 \zeta f^{\prime}(1 / \zeta)}-\frac{1}{2}\right]\right. \\
& +\frac{1}{1-\zeta / z}\left[\frac{f^{\prime \prime}(1 / \zeta)}{2 \zeta f^{\prime}(1 / \zeta)}+\frac{f^{\prime \prime \prime}(1 / \zeta)}{6 \zeta^{2} f^{\prime}(1 / \zeta)}\right. \\
& \left.\left.+\frac{f^{\prime \prime}(1 / \zeta)^{2}}{4 \zeta^{2} f^{\prime}(1 / \zeta)^{2}}-\frac{f^{\prime}(1 / \zeta)^{2}}{\zeta^{2} f(1 / \zeta)^{2}}\right]\right\} \\
& -e^{-2 i \theta} z g^{\prime}(z) B\left(\frac{1}{z}\right) \\
& =-e^{-2 i \theta} g^{\prime}(z)\left\{\frac{1}{\bar{\zeta}^{3} z^{2}\left[1-(\bar{\zeta} z)^{-1}\right]^{3}}\right. \\
& +\frac{1}{z\left[1-(\bar{\zeta} z)^{-1}\right]^{2}} \overline{\left[\frac{1}{2 \zeta^{2}}-\frac{f^{\prime \prime}(1 / \zeta)}{2 \zeta^{3} f^{\prime}(1 / \zeta)}\right]} \\
& \left.+\frac{1}{1-(\bar{\zeta} z)^{-1}}\left[\frac{f^{\prime \prime}(1 / \zeta)}{2 \zeta^{2} f^{\prime}(1 / \zeta)}+\frac{f^{\prime \prime \prime}(1 / \zeta)}{6 \zeta^{3} f^{\prime}(1 / \zeta)}+\frac{f^{\prime \prime}(1 / \zeta)^{2}}{4 \zeta^{3} f^{\prime}(1 / \zeta)^{2}}-\frac{f^{\prime}(1 / \zeta)^{2}}{\zeta^{3} f(1 / \zeta)^{2}}\right]\right\},
\end{aligned}
$$

and

$$
\begin{aligned}
-z g^{\prime}(z) C\left(\frac{1}{z}\right)=-z g^{\prime}(z)\left\{\frac{1}{\left(|\zeta|^{2}-1\right)(1-\zeta / z)^{2}}\right. & \\
& -\frac{1}{1-\zeta / z}\left[\frac{2}{|\zeta|^{2}-1}+\frac{1}{\left(|\zeta|^{2}-1\right)^{2}}\right] \\
& -\frac{1}{\bar{\zeta}^{2}\left(|\zeta|^{2}-1\right)\left[1-(\bar{\zeta} z)^{-1}\right]^{2} z^{2}} \\
& \left.-\frac{1}{z\left[1-(\bar{\zeta} z)^{-1}\right]}\left[\frac{2}{\bar{\zeta}\left(|\zeta|^{2}-1\right)}+\frac{1}{\bar{\zeta}\left(|\zeta|^{2}-1\right)^{2}}\right]\right\}
\end{aligned}
$$

Techniques analogous to those used previously then allow us to obtain the following:

$$
\begin{gathered}
\operatorname{Re}\left\{-z g^{\prime}(z) \psi_{1}\left(\frac{1}{z}\right)\right\}_{-n}=-\operatorname{Re}\left\{\frac{n e^{-2 i \theta}}{2 \bar{\zeta}^{n+1}}\right\}, \\
\operatorname{Re}\left\{-z g^{\prime}(z) \psi_{2}\left(\frac{1}{z}\right)\right\}_{-n}=-\operatorname{Re}\left\{\sum_{k=0}^{n} \frac{\zeta^{n-k}}{\bar{\zeta}^{k+1}}\right\}, \\
\operatorname{Re}\left\{-z g^{\prime}(z) \psi_{3}\left(\frac{1}{z}\right)\right\}_{-n}=\operatorname{Re}\left\{\frac{n}{n+1} e^{2 i \theta}-\frac{n+2}{2} \xi^{n+1} e^{2 i \theta}\right\},
\end{gathered}
$$




$$
\operatorname{Re}\left\{-z g^{\prime}(z) \psi_{4}\left(\frac{1}{z}\right)\right\}_{-n}=\operatorname{Re}\left\{-z g^{\prime}(z) \psi_{5}\left(\frac{1}{z}\right)\right\}_{-n}=0
$$

(36) $\operatorname{Re}\left\{-z g^{\prime}(z) \psi_{7}\left(\frac{1}{z}\right)\right\}_{-n}=\operatorname{Re}\left\{\zeta^{2} g^{\prime}(\zeta)^{2} \sum_{k=0}^{n-1} \frac{(n-k+1) g(\zeta)^{n-2-k}}{\bar{\zeta}^{k+1}}\right\}$,

(37) $\operatorname{Re}\left\{-z g^{\prime}(z) \psi_{8}\left(\frac{1}{z}\right)\right\}_{-n}=\operatorname{Re}\left\{e^{2 i \theta} g^{\prime}(\zeta)^{2} \sum_{k=0}^{n}(n-k+2) g(\zeta)^{n-k-1} \zeta^{k+2}\right\}$,

$$
\begin{aligned}
& \operatorname{Re}\left\{-e^{2 i \theta} z g^{\prime}(z) A\left(\frac{1}{z}\right)\right\}_{-n} \\
&=-\operatorname{Re}\left\{e ^ { 2 i \theta } \left[\frac{(n+2)^{2}}{2} \zeta^{n+1}-\frac{n}{n+1}+\frac{(n+3) \zeta^{n} f^{\prime \prime}(1 / \zeta)}{2 f^{\prime}(1 / \zeta)}\right.\right. \\
& \quad-\frac{2 n f^{\prime \prime}(1 / \zeta)}{(n+1) \zeta f^{\prime}(1 / \zeta)}+\frac{\zeta^{n-1} f^{\prime \prime \prime}(1 / \zeta)}{6 f^{\prime}(1 / \zeta)}+\frac{\zeta^{n-1} f^{\prime \prime}(1 / \zeta)^{2}}{4 f^{\prime}(1 / \zeta)^{2}}-\frac{\zeta^{n-1} f^{\prime}(1 / \zeta)^{2}}{f(1 / \zeta)^{2}} \\
&\left.\left.\quad-\frac{n f^{\prime \prime \prime}(1 / \zeta)}{3(n+1) \zeta^{2} f^{\prime}(1 / \zeta)}+\frac{2 n f^{\prime}(1 / \zeta)^{2}}{(n+1) \zeta^{2} f(1 / \zeta)^{2}}-\frac{n f^{\prime \prime}(1 / \zeta)^{2}}{2(n+1) \zeta^{2} f^{\prime}(1 / \zeta)^{2}}\right]\right\},
\end{aligned}
$$

(39)

$$
\begin{aligned}
\operatorname{Re}\{ & \left.-e^{-2 i \theta} z g^{\prime}(z) B\left(\frac{1}{z}\right)\right\}_{-n} \\
& =-\operatorname{Re}\left\{e^{-2 i \theta}\left[\frac{n^{2}}{2 \zeta^{n+1}}-\frac{(n-1) f^{\prime \prime}(1 / \zeta)}{2 \zeta^{n+2} f^{\prime}(1 / \zeta)}+\frac{f^{\prime \prime \prime}(1 / \zeta)}{6 \zeta^{n+3} f^{\prime}(1 / \zeta)}+\frac{f^{\prime \prime}(1 / \zeta)^{2}}{4 \zeta^{n+3} f^{\prime}(1 / \zeta)^{2}}-\frac{f^{\prime}(1 / \zeta)^{2}}{\zeta^{n+3} f(1 / \zeta)}\right]\right\},
\end{aligned}
$$

and

(40)

$$
\begin{aligned}
\operatorname{Re}\left\{-z g^{\prime}(z) C\left(\frac{1}{z}\right)\right\}_{-n}=\operatorname{Re}\{ & -\frac{2 n}{(n+1)\left(|\zeta|^{2}-1\right)}-\frac{n \zeta^{n+1}}{|\zeta|^{2}-1}+\frac{\zeta^{n+1}}{\left(|\zeta|^{2}-1\right)^{2}} \\
& \left.-\frac{2 n}{(n+1)\left(|\zeta|^{2}-1\right)^{2}}+\frac{n+2}{\bar{\zeta}^{n+1}\left(|\zeta|^{2}-1\right)}+\frac{1}{\bar{\zeta}^{n+1}\left(|\zeta|^{2}-1\right)^{2}}\right\} .
\end{aligned}
$$

Adding the right-hand sides of equations (24)-(40), we obtain the second-variational formula

$$
\operatorname{Re}\left\{V_{2}(z)\right\}_{-n}=\operatorname{Re}\left\{e^{2 i \theta} R(\zeta)+e^{-2 i \theta} S(\zeta)+T(\zeta)\right\},
$$


where

$$
\begin{aligned}
R(\zeta)= & +n \zeta^{4} g^{\prime}(\zeta)^{4} g(\zeta)^{n-3}-\frac{(n+1)(n+2) \zeta^{n+1}}{2} \\
& +g^{\prime}(\zeta)^{2} \sum_{k=0}^{n-1}(n-k) g(\zeta)^{n-k-1} \zeta^{k+2}+\frac{2 f^{\prime \prime}(1 / \zeta)}{\zeta f^{\prime}(1 / \zeta)}+\frac{f^{\prime \prime \prime}(1 / \zeta)}{3 \zeta^{2} f^{\prime}(1 / \zeta)} \\
& +\frac{f^{\prime \prime}(1 / \zeta)^{2}}{2 \zeta^{2} f^{\prime}(1 / \zeta)^{2}}-\frac{2 f^{\prime}(1 / \zeta)^{2}}{\zeta^{2} f(1 / \zeta)^{2}}-\frac{(n+3) f^{\prime \prime}(1 / \zeta) \zeta^{n}}{2 f^{\prime}(1 / \zeta)} \\
& -\frac{\zeta^{n-1} f^{\prime \prime \prime}(1 / \zeta)}{6 f^{\prime}(1 / \zeta)}-\frac{\zeta^{n-1} f^{\prime \prime}(1 / \zeta)^{2}}{4 f^{\prime}(1 / \zeta)^{2}}+\frac{\zeta^{n-1} f^{\prime}(1 / \zeta)^{2}}{f(1 / \zeta)^{2}}+\frac{1}{\zeta^{n+1}}, \\
& \quad-\frac{(n-1) n}{2(\zeta)}=-\frac{(n-1) f^{\prime \prime}(1 / \zeta)}{2 \zeta^{n+2} f^{\prime}(1 / \zeta)} \\
& -\frac{f^{\prime \prime \prime}(1 / \zeta)}{6 \zeta^{n+3} f^{\prime}(1 / \zeta)}-\frac{f^{\prime \prime}(1 / \zeta)^{2}}{4 \zeta^{n+3} f^{\prime}(1 / \zeta)^{2}}+\frac{f^{\prime}(1 / \zeta)^{2}}{\zeta^{n+3} f(1 / \zeta)^{2}},
\end{aligned}
$$

and

$$
\begin{aligned}
T(\zeta)= & \zeta^{2} g^{\prime}(\zeta)^{2} \sum_{k=0}^{n-2} \frac{(n-k-1) g(\zeta)^{n-2-k}}{\bar{\zeta}^{k+1}}+\sum_{k=0}^{n-1} \frac{\zeta^{n-k}}{\bar{\zeta}^{k+1}}-\frac{2}{|\zeta|^{2}-1}-\frac{2}{\left(|\zeta|^{2}-1\right)^{2}} \\
& +\frac{n+2}{\bar{\zeta}^{n+1}\left(|\zeta|^{2}-1\right)}+\frac{1}{\bar{\zeta}^{n+1}\left(|\zeta|^{2}-1\right)^{2}}-\frac{n \zeta^{n+1}}{|\zeta|^{2}-1}+\frac{\zeta^{n+1}}{\left(|\zeta|^{2}-1\right)^{2}}
\end{aligned}
$$

Since a number and its complex conjugate have the same real part, $\operatorname{Re}\left\{e^{-2 i \theta} S(\zeta)\right\}=$ $\operatorname{Re}\left\{e^{2 i \theta} \overline{S(\zeta)}\right\}$. Hence we can find real numbers $\theta_{+}(\zeta)$ and $\theta_{-}(\zeta)$ so that

$$
\operatorname{Re}\left\{V_{2}(z)\right\}_{-n}= \begin{cases}|R(\zeta)+\overline{S(\zeta)}|+\operatorname{Re}\{T(\zeta)\}, & \text { if } \theta=\theta_{+}(\zeta), \\ -|R(\zeta)+\overline{S(\zeta)}|+\operatorname{Re}\{T(\zeta)\}, & \text { if } \theta=\theta_{-}(\zeta)\end{cases}
$$

Let $\eta=e^{i \beta}$, where $\eta^{n+1}=-1$, and write $\zeta=\rho e^{i \beta}$. Making the substitutions

$$
\begin{aligned}
g(\zeta)=\frac{\left(\zeta^{n+1}+1\right)^{2 /(n+1)}}{\zeta}, \quad g^{\prime}(\zeta)=\frac{\zeta^{n+1}-1}{\zeta^{2}\left(\zeta^{n+1}+1\right)^{1-2 /(n+1)}}, \\
f\left(\frac{1}{\zeta}\right)=\frac{\zeta}{\left(\zeta^{n+1}+1\right)^{2 /(n+1)},} \\
f^{\prime}\left(\frac{1}{\zeta}\right)=\frac{\zeta^{2}\left(\zeta^{n+1}-1\right)}{\left(\zeta^{n+1}+1\right)^{1+2 /(n+1)}}, \\
f^{\prime \prime}\left(\frac{1}{\zeta}\right)=\frac{2 \zeta^{3}\left[1-(n+2) \zeta^{n+1}\right]}{\left(\zeta^{n+1}+1\right)^{2+2 /(n+1)}},
\end{aligned}
$$


and

$$
f^{\prime \prime \prime}\left(\frac{1}{\zeta}\right)=-\frac{2 \zeta^{4}\left[3-\left(n^{2}+8 n+9\right) \zeta^{n+1}+n(n+2) \zeta^{2 n+2}\right]}{\left(\zeta^{n+1}+1\right)^{3+2 /(n+1)}}
$$

in the expressions for $R(\zeta), S(\zeta)$, and $T(\zeta)$, it can be shown that with the choices of $\theta$ indicated,

$$
\begin{aligned}
\operatorname{Re}\left\{V_{2}(z)\right\}_{-n}=\left|\zeta^{n+1}+1\right|^{-2}[ \pm & \frac{(n+1)\left(\zeta^{n+1}-1\right)^{4}}{\zeta^{n+1}}-\frac{\left[1-(n+2) \zeta^{n+1}\right]^{2}}{\zeta^{n+1}} \\
& \left.+\frac{\left[3-\left(n^{2}+8 n+9\right) \zeta^{n+1}+n(n+2) \zeta^{2 n+2}\right]\left(\zeta^{n+1}-1\right)}{3 \zeta^{n+1}} \mid\right] \\
& +\operatorname{Re}\left\{\frac{\left(\zeta^{n+1}-1\right)^{2}}{\zeta^{n+1}\left(|\zeta|^{2}-1\right)^{2}}\right\}+o\left(\left|\zeta^{n+1}+1\right|^{-2}\right),
\end{aligned}
$$

where the choice of sign in the first term depends on whether $\theta$ is $\theta_{+}(\zeta)$ or $\theta_{-}(\zeta)$. Clearly then,

$$
\lim _{\rho \rightarrow 1+}\left|\zeta^{n+1}+1\right|^{2} \operatorname{Re}\left\{V_{2}(z)\right\}_{-n}= \begin{cases}\frac{1}{3}\left|7 n^{2}-10 n+3\right|-(n+1)^{2}, & \text { if } \theta=\theta_{+}(\zeta), \\ -\frac{1}{3}\left|7 n^{2}-10 n+3\right|-(n+1)^{2}, & \text { if } \theta=\theta_{-}(\zeta) .\end{cases}
$$

Since $7 n^{2}-10 n+3 \geqslant 0$ for all $n \geqslant 1$, we have established that

$$
\lim _{\rho \rightarrow 1+}\left|\zeta^{n+1}+1\right|^{2} \operatorname{Re}\left\{V_{2}(z)\right\}_{-n}=\frac{4}{3}\left(n^{2}-4 n\right), \quad \text { if } \theta=\theta_{+}(\zeta)
$$

and

$$
\lim _{\rho \rightarrow 1+}\left|\zeta^{n+1}+1\right|^{2} \operatorname{Re}\left\{V_{2}(z)\right\}_{-n}=-\frac{2}{3}\left(5 n^{2}-2 n+3\right), \quad \text { if } \theta=\theta_{-}(\zeta) .
$$

Since $n^{2}-4 n>0$ for $n>4$, equation (41) shows that, for some choice of $\zeta$ and $\theta$, $\operatorname{Re}\left\{V_{2}(z)\right\}_{-n}>0$. Since $5 n^{2}-2 n+38>0$ for all $n$, equation (42) shows that for some choice of $\zeta$ and $\theta, \operatorname{Re}\left\{V_{2}(z)\right\}_{-n}<0$. Hence we have established (12).

The author wishes to thank Professor G. Schober for a valuable conversation and Professor P. L. Duren for his guidance and inspiration. In a forthcoming paper, Chang, Schiffer, and Schober develop a new form of the second variation to give another proof of the theorem in $\S 3$.

\section{REFERENCES}

1. I. E. Bazilevich, Supplement to the papers "Zum Koeffizientenproblem der schlichten Funktionen" and "Sur les théorèmes de Koebe-Bieberbach", Mat. Sb. 2 (44) (1937), 689-698. (Russian)

2. L. Bieberbach, Über einige Extremalprobleme im Gebiete der konformen Abbildung, Math. Ann. 77 (1915-1916), 153-172.

3. __ Über die Koeffizienten derjenigen Potenzreihen, welche eine schlichte Abbildung des Einheitskreises vermitteln, S.-B. Preuss. Akad. Wiss. (1916), 940-955.

4. J. Clunie, On schlicht functions, Ann. of Math. (2) 69 (1959), 511-519.

5. P. L. Duren, Coefficients of meromorphic schlicht functions, Proc. Amer. Math. Soc. 28 (1971), $169-172$

6. , Univalent functions, Springer-Verlag, Berlin and New York (to appear). 
7. G. M. Goluzin, Geometric theory of functions of a complex variable, "Nauka", Moscow, 1966; English transl., Transl. Math. Monographs, vol. 26, Amer. Math. Soc., Providence, R. I., 1969.

8. T. H. Gronwall, Some remarks on conformal representation, Ann. of Math. (2) 16 (1914-1915), $72-76$.

9. J. A. Jenkins, On certain coefficients of univalent functions. II, Trans. Amer. Math. Soc. 96 (1960), $534-545$

10. Yoshihisa Kubota, $A$ coefficient inequality for certain meromorphic univalent functions, Kōdai Math. Sem. Rep. 26 (1974/75), 85-94.

11. On the fourth coefficient of meromorphic univalent functions, Kōdai Math. Sem. Rep. 26 (1974/75), 267-288.

12. Ch. Pommerenke, On a variational method for univalent functions, Michigan Math. J. 17 (1970), 1-3.

13. __ Univalent functions (with a chapter on quadratic differentials by G. Jensen), Vandenhoeck and Ruprecht, Göttingen, 1975.

14. M. Schiffer, Sur un problème d'extrémum de la représentation conforme, Bull. Soc. Math. France 66 (1938), 48-55.

Department of Mathematics, U. S. Naval Academy, Annapolis, Maryland 21402 